

\title{
Modified Rayleigh Conjecture and Applications *广
}

\author{
A.G. Ramm \\ LMA/CNRS, Marseille 14302, cedex 20, France \\ and Mathematics Department, Kansas State University, \\ Manhattan, KS 66506-2602, USA \\ ramm@math.ksu.edu
}

\begin{abstract}
Modified Rayleigh conjecture (MRC) in scattering theory is proposed and justified. MRC allows one to develop numerical algorithms for solving direct scattering problems related to acoustic wave scattering by soft and hard obstacles of arbitrary shapes. It gives an error estimate for solving the direct scattering problem. It suggests a numerical method for finding the shape of a starshaped obstacle from the scattering data.
\end{abstract}

\section{Introduction}

Consider a bounded domain $D \subset \mathbb{R}^{n}, n=3$ with a boundary $S$. The exterior domain is $D^{\prime}=\mathbb{R}^{3} \backslash D$. Assume that $S$ is smooth and starshaped, that is, its equation can be written as

$$
r=f(\alpha)
$$

where $\alpha \in S^{2}$ is a unit vector and $S^{2}$ denotes the unit sphere in $R^{3}$. Smoothness of $S$ is used in (4.6) below. For solving the direct scattering problem by the method described in the beginning of Section 2, the boundary $S$ can be Lipschitz. The acoustic wave scattering problem by a soft obstacle $D$ consists in finding the (unique) solution to the problem (1.2)-(1.3):

$$
\begin{gathered}
\left(\nabla^{2}+k^{2}\right) u=0 \text { in } D^{\prime}, \quad u=0 \text { on } S, \\
u=u_{0}+A\left(\alpha^{\prime}, \alpha\right) \frac{e^{i k r}}{r}+o\left(\frac{1}{r}\right), \quad r:=|x| \rightarrow \infty, \quad \alpha^{\prime}:=\frac{x}{r} .
\end{gathered}
$$

*key words: Rayleigh hypothesis, scattering by obstacles, inverse scattering

$\dagger$ AMS subject classification: 35R30 
Here $u_{0}:=e^{i k \alpha \cdot x}$ is the incident field, $A\left(\alpha^{\prime}, \alpha\right)$ is called the scattering amplitude, its $\mathrm{k}$-dependence is not shown, $k>0$ is the wavenumber. Denote

$$
A_{\ell}(\alpha):=\int_{S^{2}} A\left(\alpha^{\prime}, \alpha\right) \overline{Y_{\ell}\left(\alpha^{\prime}\right)} d \alpha^{\prime}
$$

where $Y_{\ell}(\alpha)$ are the orthonormal spherical harmonics, $Y_{\ell}=Y_{\ell m},-\ell \leq m \leq \ell$. Let $h_{\ell}(r)$ be the spherical Hankel functions, normalized so that $h_{\ell}(r) \sim \frac{e^{i k r}}{r}$ as $r \rightarrow+\infty$. Let the ball $B_{R}:=\{x:|x| \leq R\}$ contain $D$.

In the region $r>R$ the solution to (1.2) - (1.3) is:

$$
u(x, \alpha)=e^{i k \alpha \cdot x}+\sum_{\ell=0}^{\infty} A_{\ell}(\alpha) \psi_{\ell}, \quad \psi_{\ell}:=Y_{\ell}\left(\alpha^{\prime}\right) h_{\ell}(k r), \quad r>R, \quad \alpha^{\prime}=\frac{x}{r},
$$

summation includes summation with respect to $m,-\ell \leq m \leq \ell$, and $A_{\ell}(\alpha)$ are defined in (1.4).

Rayleigh conjecture $(\mathrm{RC})$ : the series (1.5) converges up to the boundary $S$ (originally $\mathrm{RC}$ dealt with periodic structures, gratings). This conjecture is wrong [1], [3], [4]. For example, if $n=2$ and $D$ is an ellipse, then the series analogous to (1.5) converges in the region $r>a$, where $2 a$ is the distance between the foci of the ellipse [1]. In the engineering literature there are numerical algorithms, based on the Rayleigh conjecture. Our aim is to give a formulation of a modified Rayleigh conjecture (MRC) which is correct and can be used in numerical solution of the direct and inverse scattering problems. We discuss the Dirichlet condition but similar argument is applicable to the Neumann boundary condition, corresponding to acoustically hard obstacles.

Fix $\epsilon>0$, an arbitrary small number.

Lemma 1.1. There exist $L=L(\epsilon)$ and $c_{\ell}=c_{\ell}(\epsilon)$ such that

$$
\left\|u_{0}+\sum_{\ell=0}^{L(\epsilon)} c_{\ell}(\epsilon) \psi_{l}\right\|_{L^{2}(S)} \leq \epsilon
$$

If (1.6) and the boundary condition (1.2) hold, then

$$
\left\|v_{\epsilon}-v\right\|_{L^{2}(S)} \leq \epsilon, \quad v_{\epsilon}:=\sum_{\ell=0}^{L(\epsilon)} c_{\ell}(\epsilon) \psi_{l} .
$$

Lemma 1.2. If (1.7) holds then

$$
\left\|v_{\epsilon}-v\right\|=O(\epsilon) \quad \epsilon \rightarrow 0
$$

where $\|\cdot\|:=\|\cdot\|_{H_{l o c}^{m}\left(D^{\prime}\right)}+\|\cdot\|_{L^{2}\left(D^{\prime} ;(1+|x|)^{-\gamma}\right)}, \gamma>1, m>0$ is an arbitrary integer, and $H^{m}$ is the Sobolev space. 
In particular, (1.8) implies

$$
\left\|v_{\epsilon}-v\right\|_{L^{2}\left(S_{R}\right)}=O(\epsilon) \quad \epsilon \rightarrow 0 .
$$

Lemma 1.3. One has:

$$
c_{\ell}(\epsilon) \rightarrow A_{\ell}(\alpha) \forall \ell, \quad \epsilon \rightarrow 0 .
$$

The modified Rayleigh conjecture (MRC) is formulated as a theorem, which follows from the above three lemmas:

Theorem 1 (MRC): For an arbitrary small $\epsilon>0$ there exist $L(\epsilon)$ and $c_{\ell}(\epsilon), 0 \leq$ $\ell \leq L(\epsilon)$, such that (1.6), (1.8) and (1.10) hold.

The difference between RC and MRC is: (1.7) does not hold if one replaces $v_{\epsilon}$ by $\sum_{\ell=0}^{L} A_{\ell}(\alpha) \psi_{\ell}$, and let $L \rightarrow \infty$ (instead of letting $\epsilon \rightarrow 0$ ).

For the Neumann boundary condition one minimizes $\left\|\frac{\partial\left[u_{0}+\sum_{\ell=0}^{L} c_{\ell} \psi_{\ell}\right]}{\partial N}\right\|_{L^{2}(S)}$ with respect to $c_{\ell}$. Analogs of Lemmas 1.1-1.3 are valid and their proofs are essentially the same.

In Section 2 we discuss the usage of MRC in solving the direct scattering problem, in Section 3 its usage in solving the inverse scattering problem, and in Section 4 proofs are given.

\section{Direct scattering problem and MRC.}

The direct problem consists in finding the scattered field $v$ given $S$ and $u_{0}$. To solve it using MRC, fix a small $\epsilon>0$ and find $L(\epsilon)$ and $c_{\ell}(\epsilon)$ such that (1.6) holds. This is possible by Lemma 1.1 and can be done numerically by minimizing $\left\|u_{0}+\sum_{0}^{L} c_{\ell} \psi_{\ell}\right\|_{L^{2}(S)}:=$ $\phi\left(c_{1}, \ldots ., c_{L}\right)$. If the minimum of $\phi$ is larger than $\epsilon$, then increase $L$ and repeat the minimization. Lemma 1.1 guarantees the existence of such $L$ and $c_{\ell}$ that the minimum is less than $\epsilon$. Choose the smallest $L$ for which this happens and define $v_{\epsilon}:=\sum_{\ell=0}^{L} c_{\ell} \psi_{\ell}(x)$. Then $v_{\epsilon}$ is the approximate solution to the direct scattering problem with the accuracy $O(\epsilon)$ in the norm $\|\cdot\|$ by Lemma 1.2.

In [6] representations of $v$ and $v_{\epsilon}$ are proposed, which greatly simplified minimization of $\phi$. Namely, let $\Psi_{\ell}$ solve problem

$$
\left(\nabla^{2}+k^{2}\right) \Psi_{\ell}=0 \text { in } D^{\prime}, \quad \Psi_{\ell}=f_{\ell} \text { on } S
$$

and $\Psi_{\ell}$ satisfies the radiation condition. Here $\left\{f_{\ell}\right\}_{\ell \geq 0}$ is an arbitrary orthonormal basis of $L^{2}(S)$. Denote

$$
v(x):=\sum_{\ell=0}^{\infty} c_{\ell} \Psi_{\ell}(x), \quad u(x):=u_{0}+v(x), \quad c_{l}:=\left(-u_{0}, f_{\ell}\right)_{L^{2}(S)} .
$$

The series (2.2) on $S$ is a Fourier series which converges in $L^{2}(S)$. It converges pointwise in $D^{\prime}$ by the argument given in the proof of Lemma 1.2. A possible choice of $f_{\ell}$ for star-shaped $S$ is $f_{\ell}=Y_{\ell} / \sqrt{w}$ where $w:=d S / d \alpha$. Here $d S$ and $d \alpha$ are respectively the elements of the surface areas of the surface $S$ and of the unit sphere $S^{2}$. 


\section{Inverse scattering problem and MRC.}

Inverse obstacle scattering problems IOSPa) and IOSPb) consist of finding $S$ and the boundary condition on $S$ from the knowledge of:

IOSPa): the scattering data $A\left(\alpha^{\prime}, \alpha, k_{0}\right)$ for all $\alpha^{\prime}, \alpha \in S^{2}, k=k_{0}>0$ being fixed, or,

$\mathrm{IOSPb}): A\left(\alpha^{\prime}, \alpha_{0}, k\right)$, known for all $\alpha^{\prime} \in S^{2}$ and all $k>0, \alpha=\alpha_{0} \in S^{2}$ being fixed.

Uniqueness of the solution to IOSPa) is proved by A.G.Ramm (1985) for the Dirichlet, Neumann and Robin boundary conditions, and of IOSPb) by M.Schiffer (1964), who assumed a priori the Dirichlet boundary condition. The proofs are given in [4]. A.G.Ramm has also proved that not only $S$ but the boundary condition as well is uniquely defined by the above data in both cases, and gave stability estimates for the solution to IOSP [9]. Later he gave a different method of proof of the uniqueness theorems for these problems which covered the rough boundaries (Lipschitz and much rougher boundaries: the ones with finite perimeter [8], see also [10]. In [11] the uniqueness theorem for the solution of inverse scattering problem is proved for a wide class of transmission problems. It is proved that not only the discontinuity surfaces of the refraction coefficient but also the coefficient itself inside the body and the boundary conditions across these surfaces are uniquely determined by the fixed-frequency scattering data. For any strictly convex, smooth, reflecting obstacle $D$ analytical formulas for finding $S$ from the high-frequency asymptotics of the scattering amplitude are proposed by A.G.Ramm, who gave error estimates of his inversion formula also [4]. The uniqueness theorems in the above references hold if the scattering data are given not for all $\alpha^{\prime}, \alpha \in S^{2}$, but only for $\alpha^{\prime}$ and $\alpha$ in arbitrary small solid angles, i.e., in arbitrary small open subsets of $S^{2}$. The inverse scattering problem with the data $\alpha^{\prime} \in S^{2}, k=k_{0}$ and $\alpha=\alpha_{0}$ being fixed, is open. If a priori one knows that $D$ is sufficiently small, so that $k_{0}>0$ is not a Dirichlet eigenvalue of the laplacian in $D$, then uniqueness of the solution with the above non-overdetermined data holds (by the usual argument [4]). There are many parameter-fitting schemes for solving IOSP, [13], see also [5].

Let us describe a new such scheme, based on MRC, its idea is similar to the one in [7]. Suppose that the scattered field $v$ is observed on a sphere $S_{R}$. Calculate $c_{\ell}:=$ $\left(v, Y_{\ell}\right)_{L^{2}\left(S^{2}\right)} / h_{\ell}(k R)$. If $v$ is known exactly, then $c_{\ell}=A_{\ell}(\alpha)$. If $v_{\delta}$ are noisy data, $\| v-$ $v_{\delta} \|_{L^{2}\left(S_{R}\right)} \leq \delta$, then $c_{\ell}=c_{\ell \delta}$. Choose some $L$, say $L=5$, and find $r=r\left(\alpha^{\prime}\right)$ as a positive root of the equation $u_{0}+v_{L}:=e^{i k \alpha \cdot \alpha^{\prime} r}+\sum_{\ell=0}^{L} c_{\ell \delta} \psi_{\ell}\left(k r, \alpha^{\prime}\right):=p\left(r, \alpha^{\prime}, \alpha, k\right)=0$. Here $\alpha^{\prime}$ and $k>0$ are fixed, and we are looking for the root $r=r\left(\alpha^{\prime}\right)$ which is positive and stable under changes of $k$ and $\alpha$. In practice equation $p\left(r, \alpha^{\prime}, \alpha, k\right)=0$ may have no such root, the root may have small imaginary part. If for the chosen $L$ such a root (that is, a root which is positive, or has a small imaginary part, and stable with respect to changes of $k$ and $\alpha$ ) is not found, then increase $L$, and/or decrease $L$, and repeat the search of the root. Stop the search at a smallest $L$ for which such a root is found. The MRC justifies this method: for a suitable $L$ the function $p\left(r, \alpha^{\prime}, \alpha, k\right)$ is approximately equals zero on $S$, that is, for $r=r\left(\alpha^{\prime}\right)$, and this $r\left(\alpha^{\prime}\right)$ does not depend on $k$ and $\alpha$. Moreover, 
by the uniqueness theorem for IOSPa) and IOSPb) there is only one such $r=r\left(\alpha^{\prime}\right)$. Numerically one expects to find a root of the equation $p\left(r, \alpha^{\prime}, k\right)=0$ which is close to positive semiaxis $r>0$ and stable with respect to changes of $k$ and $\alpha$.

If one uses the above scheme for solving the inverse scattering problem for an acoustically hard body (the Neumann boundary condition on $S$ ), then one gets not a transcendental equation $p\left(r, \alpha^{\prime}, \alpha, k\right)=0$ for finding the equation of $S, r=r\left(\alpha^{\prime}\right)$, but a differential equation for $r=r\left(\alpha^{\prime}\right)$, which comes from the equation $\frac{\partial p\left(r, \alpha^{\prime}, \alpha, k\right)}{\partial N}=0$ at $r=r\left(\alpha^{\prime}\right)$. One has to write the normal derivative on $S$ in spherical coordinates and then substitute $r=r\left(\alpha^{\prime}\right)$ into the result to get a differential equation for the unknown function $r=r\left(\alpha^{\prime}\right)$. For example, if $n=2$ (the two-dimensional case), then the role of $\alpha^{\prime}$ plays the polar angle $\varphi^{\prime}$ and the equation for $r=r\left(\varphi^{\prime}\right)$ takes the form $\frac{d r}{d \varphi^{\prime}}=\left.\left(r^{2} \frac{d p}{d r} / \frac{d p}{d \varphi^{\prime}}\right)\right|_{r=r\left(\varphi^{\prime}\right)}$.

\section{Proofs.}

Proof of Lemma 1.1. This Lemma follows from the results in [4], (p.162, Lemma 1). Proof of Lemma 1.2. By Green's formula one has

$$
v_{\epsilon}(x)=-\int_{S} v_{\epsilon}(s) G_{N}(x, s) d s, \quad\left\|v_{\epsilon}(s)+u_{0}\right\|_{L^{2}(S)}<\epsilon,
$$

where $G$ is the Dirichlet Green's function of the Laplacian in $D^{\prime}$ :

$$
\begin{gathered}
\left(\nabla^{2}+k^{2}\right) G=-\delta(x-y) \text { in } D^{\prime}, \quad G=0 \text { on } S, \\
\lim _{r \rightarrow \infty} \int_{|x|=r}\left|\frac{\partial G}{\partial|x|}-i k G\right|^{2} d s=0
\end{gathered}
$$

From (4.1) one gets (1.3) with $H_{l o c}^{m}\left(D^{\prime}\right)$-norm immediately by the Cauchy inequality, and with the weighted norm from the estimate

$$
\left|G_{N}(x, s)\right| \leq \frac{c}{1+|x|}, \quad|x| \geq R
$$

and from local elliptic estimates for $w_{\epsilon}:=v_{\epsilon}-v$, which imply that

$$
\left\|w_{\epsilon}\right\|_{L^{2}\left(B_{R} \backslash D\right)} \leq c \epsilon .
$$

Let us recall the elliptic estimate we use. Let $D_{R}^{\prime}:=B_{R} \backslash D, S_{R}$ be the boundary of $B_{R}$, and choose $R$ such that $k^{2}$ is not a Dirichlet eigenvalue of $-\Delta$ in $D_{R}^{\prime}$. The elliptic estimate we have used is ([2, p.189):

$$
\left\|w_{\epsilon}\right\|_{H^{m}\left(D_{R}^{\prime}\right)} \leq c\left[\left\|\left(\Delta+k^{2}\right) w_{\epsilon}\right\|_{H^{m-2}\left(D_{R}^{\prime}\right)}+\left\|w_{\epsilon}\right\|_{H^{m-0.5}\left(S_{R}\right)}+\left\|w_{\epsilon}\right\|_{H^{m-0.5}(S)}\right] .
$$

Take $m=0.5$ in (4.7), use the equation $\left(\Delta+k^{2}\right) w_{\epsilon}=0$ in $D^{\prime}$, the estimate $\left\|w_{\epsilon}\right\|_{H^{m}\left(S_{R}\right)}=$ $O(\epsilon)$, proved above, the estimate $\left\|w_{\epsilon}\right\|_{H^{0}(S)}=O(\epsilon)$, and get (1.8). Lemma 1.2 is proved. 
Proof of Lemma 1.3. Lemma 1.2 yields convergence of $v_{\epsilon}$ to $v$ in the norm $\|\cdot\|$. In particular, $\left\|v_{\epsilon}-v\right\|_{L^{2}\left(S_{R}\right)} \rightarrow 0$ as $\epsilon \rightarrow 0$. On $S_{R}$ one has $v=\sum_{\ell=0}^{\infty} A_{\ell}(\alpha) \psi_{\ell}$ and $v_{\epsilon}=\sum_{\ell=0}^{L(\epsilon)} c_{\ell} \psi_{\ell}$. Multiply $v_{\epsilon}\left(R, \alpha^{\prime}\right)-v\left(R, \alpha^{\prime}\right)$ by $\overline{Y_{\ell}\left(\alpha^{\prime}\right)}$, integrate over $S^{2}$ and then let $\epsilon \rightarrow 0$. The result is (1.10).

\section{References}

[1] Barantsev, R., Concerning the Rayleigh hypothesis in the problem of scattering from finite bodies of arbitrary shapes, Vestnik Lenigrad. Univ., Math., Mech., Astron., 7, (1971), 56-62.

[2] Lions, J.L., Magenes, E., Non-homogeneous boundary value problems and applications, Springer Verlag, New York, 1972.

[3] Millar, R., The Rayleigh hypothesis and a related least-squares solution to scattering problems for periodic surfaces and other scatterers, Radio Sci., 8, (1973), 785-796.

[4] Ramm, A.G., Scattering by obstacles, D.Reidel, Dordrecht, 1986, pp.1-442.

[5] Ramm, A.G., Multidimensional inverse scattering problems, Longman/Wiley, New York, 1992, pp.1-385.

[6] Ramm, A.G., Numerically efficient version of the T-matrix method, Applic. Anal., 80, N3, (2002), 3850-393.

[7] Ramm, A.G., A geometrical inverse problem, Inverse problems, 2, (1986), L19-21.

[8] Ramm, A.G., Uniqueness theorems for inverse obstacle scattering problems in Lipschitz domains, Applic. Analysis, 59, (1995), 377-383.

[9] Ramm, A.G., Stability of the solution to inverse obstacle scattering problem, J.Inverse and Ill-Posed Problems, 2, N3, (1994), 269-275.

[10] Ramm, A.G., Sammartino, M., Existence and uniqueness of the scattering solutions in the exterior of rough domains, in the book "Operator Theory and Its Applications", Amer. Math. Soc., Fields Institute Communications vol.25, pp.457-472, Providence, RI, 2000. (editors A.G.Ramm, P.N.Shivakumar, A.V. Strauss)

[11] Ramm, A.G., Pang, P., and Yan, G., A uniqueness result for the inverse transmission problem, Internat. Jour. of Appl. Math., 2, N5, (2000), 625-634.

[12] Ramm, A.G., On Rayleigh conjecture,

[13] Scotti, T., Wirgin, A., Shape reconstruction of an impenetrable body via the Rayleigh hypothesis, Inverse probl., 12, (1996), 1027-1055. 